\title{
First Test Results of a High-Speed Beam Conditions Monitor for the Atlas Experiment
}

\author{
H. Pernegger
}

\begin{abstract}
The paper presents the design and first test results of the high-speed Beam Conditions Monitor (BCM) for the ATLAS Experiment at the Large-Hadron Collider (LHC). The goal of the BCM is to monitor instantaneous rates of collision and background and detect signs of beam instabilities. The detector is based on polycrystalline Chemical-Vapor-Deposition (pCVD) diamond as active sensor material. We have chosen this detector material for its proven radiation hardness and fast signal properties. For the readout of the diamonds we developed front-end readout electronics based on high-bandwidth RF amplifiers. The signal response of the amplifier has been optimized for the ionization current signal of pCVD diamond in order to achieve a signal rise time of $\leq 1 \mathrm{~ns}$ and pulsewidth of less than $3 \mathrm{~ns}$. We present test results which were obtained on prototype assemblies in source tests and testbeam. The obtained results demonstrate the feasibility of such a detector system for the ATLAS BCM. The tests yield BCM signals with a mean rise time of 900 ps and a FWHM pulsewidth of $2.1 \mathrm{~ns}$ after $16 \mathrm{~m}$ of required transmission cable. The recorded signal amplitude distribution yields an most probable signal-to-noise ratio of 8.6:1 for single minimum-ionizing particles (MIP).
\end{abstract}

Index Terms-Beam diagnostic, diamond, high-speed electronics, Large-Hadron Collider (LHC), proton detectors, semiconductor radiation detectors.

\section{INTRODUCTION}

$\mathbf{T}$ HIS PAPER presents the design and first test results of the high-speed Beam Conditions Monitor (BCM) for the ATLAS Experiment at the Large-Hadron Collider (LHC). The $\mathrm{BCM}$ is part of the radiation and background monitoring system inside the ATLAS Inner Detector. The goal of the BCM is to:

- monitor instantaneous rates of collision and background (e.g., due to beam losses on collimators);

- provide feedback to the accelerator about the beam conditions very close to the interaction point;

- detect signs of beam instabilities and issue warning and alarm signals which can be used to protect other sensitive detector systems or trigger a beam abort.

While the last item addresses possible accident scenarios the first two measurements will provide important information during normal running conditions. The instantaneous rates of collisions and background will be measured through a

Manuscript received October 25, 2004; revised May 3, 2005. This paper was written on behalf of the ATLAS Beam Conditions Monitor Working Group, which consists of CERN, Geneva, Switzerland; Fachhochschule Wiener Neustadt/FOTEC, Wiener Neustadt, Austria; Jozef Stefan Institute, Ljubljana, Slovenia; Ohio State University, Columbus; and the University of Toronto, ON, Canada.

H. Pernegger is with the Department of Physics, CERN, Geneva, Switzerland (e-mail: heinz.pernegger@cern.ch).

Digital Object Identifier 10.1109/TNS.2005.856748

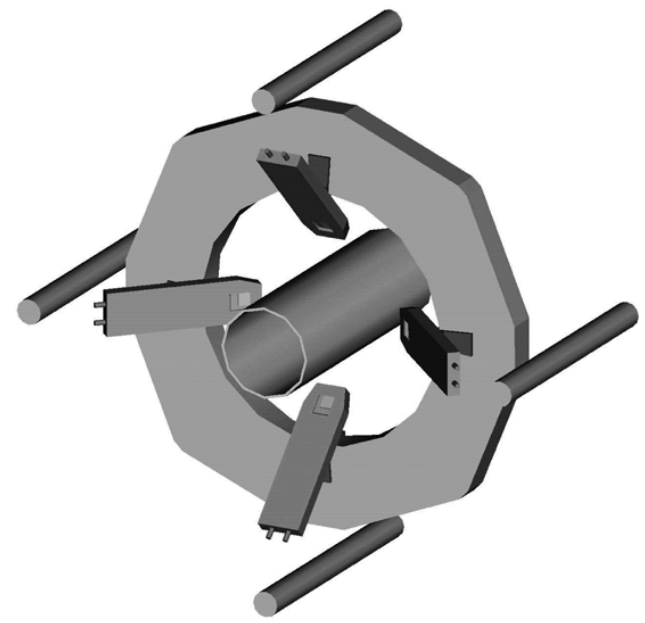

Fig. 1. Schematic illustration of four BCM stations around the beam pipe at $z=-1.84 \mathrm{~m}$. An identical setup is mounted on the $+z$-side of the pixel detector.

Time-of-Flight (TOF) measurement close to the interaction region with a TOF path of $3.68 \mathrm{~m}$. The particle flux at the location of the BCM diamond detectors during normal running is expected to be approximately one charged particle per $\mathrm{cm}^{2}$ and beam bunch crossing. We therefore require the detector electronics to provide reliable signals for single minimum-ionizing particles (MIP). For a reliable TOF measurement at each bunch crossing we aim at a signal rise time of approximately $1 \mathrm{~ns}$, an average pulse duration of less than $3 \mathrm{~ns}$, and full baseline restoration in less than $10 \mathrm{~ns}$. In addition to the TOF information, the analog pulse-height information will be used to measure the average number of charged particles in the BCM, e.g., in the case of excess beam losses, at each bunch crossing as input to the background warning and alarm logic. As the detector will operate in a high radiation environment, detectors and front-end electronics have to be radiation hard up to $10^{15}$ pions $/ \mathrm{cm}^{2}$. The detector arrangement, which is shown schematically in Fig. 1, foresees four stations at each side of the interaction point at $z= \pm 1.84 \mathrm{~m}$. The stations are mounted inside the pixel-detector support tube with an active detector acceptance area of approximately $0.5 \mathrm{~cm}^{2}$ for each station. Each station is inclined by $45^{\circ}$ with respect to the beam axis and mounted with the active detector center at a radius of $7 \mathrm{~cm}$ from the beam.

\section{THE CVD DIAMOND DETECTOR}

The detector is based on polycrystalline Chemical-Vapor-Deposition (pCVD) diamond as active sensor material, which has been developed by the CERN RD42 
collaboration [1] in cooperation with Element Six, Ltd., Berkshire, U.K. . We have chosen this detector material for its proven radiation hardness and fast signal properties. The CVD diamond, which we used for our tests, was shown to be radiation hard exceeding $10^{15}$ protons and pions per $\mathrm{cm}^{2}$ [2], [3]. The time properties of the ionization current signal are determined by the high charge carrier velocity and short charge lifetime in diamond. We operate the detector close to saturation velocity, typically at a $2 \mathrm{~V} / \mu \mathrm{m}$ drift field, and have chosen pCVD diamond with a charge lifetime in the order of nanoseconds to achieve a high and narrow current pulse. In addition pCVD diamond exhibits very low leakage current of typically less than $1 \mathrm{nA} / \mathrm{cm}^{2}$ after irradiation because of its high intrinsic resistivity and therefore does not require detector cooling.

For our tests we used several different samples of pCVD diamond with charge collection distances $\delta$ ranging from $160 \mu \mathrm{m}$ to $220 \mu \mathrm{m}$, equivalent to a mean charge signals of 5800 to 7900 electron-hole pairs for a minimum-ionizing particle [4]. The diamond charge collection distance has been measured using a collimated ${ }^{90} \mathrm{Sr}$ source with the diamond being read out through a calibrated charge-sensing amplifier [4]. $\delta$ is quoted for a drift field of $1 \mathrm{~V} / \mu \mathrm{m}$ and with the diamond being already "pumped" through exposure to a ${ }^{90} \mathrm{Sr}$ source for several hours. The pulsewidth $\tau$ can be estimated from the charge collection distance and drift velocity as

$$
\tau=\frac{2 \delta}{v_{e}+v_{h}}=1.47 \mathrm{~ns}
$$

with $\delta=220 \mu \mathrm{m}$. For the estimation, given our large operating electric field, we use the saturated electron and holes drift velocity of $v_{e}=180 \mu \mathrm{m} / \mathrm{ns}$ and $v_{h}=120 \mu \mathrm{m} / \mathrm{ns}$, respectively [5]. The diamonds measure $10 \times 10 \mathrm{~mm}^{2}$ and thicknesses range from $0.36 \mathrm{~mm}$ to $0.49 \mathrm{~mm}$. The top and bottom side of each diamond is mechanically polished and plated with evaporated $\mathrm{Cr} / \mathrm{Au}$ electrodes (annealed at $400{ }^{\circ} \mathrm{C}$ for five minutes).

\section{ThE FRONT-END ELECTRONICS}

For the readout of the BCM detector we developed a lownoise implementation of a high-speed current amplifier, which was designed for a high-speed beam diagnostic using diamonds and is described in further detail in [6]. Current amplifiers have been successfully used with diamond detectors for heavy-ion detection and can provide output signals with the time characteristics required for the BCM [7]. The BCM front-end electronics (FOTEC HFK-500, Austria) is based on a low-noise two-stage current amplifier with a total amplification of $40 \mathrm{~dB}$. We use different amplifiers with bandwidths between $500 \mathrm{MHz}$ (first stage) and $2 \mathrm{GHz}$ (second stage) to optimize the signal timing characteristics and noise performance. The input stage has a signal amplification of $22 \mathrm{~dB}$ and noise figure of $0.9 \mathrm{~dB}$ at $500 \mathrm{MHz}$. The input resistance of the amplifier is $50 \Omega$. The output of the amplifier is matched to $50 \Omega$. The analog voltage output, which is directly proportional to the detector current signal, is transmitted over $16 \mathrm{~m}$ long coaxial cables ${ }^{1}$ to the signal

${ }^{1}$ Cable type FSJ1-50A from Andrew Kommunikationssysteme AG, Bachenbülach, Switzerland. processing electronics in the nearest radiation safe zone, or, during our tests, to a digital oscilloscope for analog measurements.

During the tests the top side contact of the diamond was connected to ground through a $15 \mathrm{k} \Omega$ resistor and $\mathrm{AC}$ coupled to the preamplifier through a $1 \mathrm{nF}$ coupling capacitance. For the final assembly we consider DC coupling the diamond to the amplifier. The bottom side of the diamond is connected to the bias voltage via a $100 \mathrm{k} \Omega$ resistor.

\section{Results of Source Tests}

In order to evaluate the analog performance of the diamond-FE-electronics assembly we exposed the CVD diamond detector, while connected to the FE electronics, to a ${ }^{90} \mathrm{Sr}$ $\beta$-source. The source was mounted above the diamond with a $5 \mathrm{~mm}$ thick lead collimator in between. The collimator opening of $1 \mathrm{~mm}$ diameter is aligned to the center of the diamond. A scintillator is mounted below the diamond and provides a trigger for electrons penetrating the diamond. The acceptance area, approximately $3 \times 4 \mathrm{~mm}^{2}$, of the trigger scintillator is smaller than the diamond active area. The scope readout is triggered by the scintillator signal. The BCM is readout through a $1 \mathrm{~m}$ long coaxial cable to a LeCroy 564A digital oscilloscope with an analog bandwidth of $1 \mathrm{GHz}$ and a sampling frequency of $4 \mathrm{Gs} / \mathrm{s}$. The typical r.m.s. voltage noise on the amplifier output with the diamond connected and biased is between $500 \mu \mathrm{V}$ to $620 \mu \mathrm{V}$ for different data sets. In the offline analysis diamond pulses are selected with amplitudes exceeding $1.5 \mathrm{mV}$ unless stated otherwise. From cross-calibration with testbeam data we determined that the energy deposition in the diamond is approximately $10 \%$ above minimum ionizing in this arrangement.

The pCVD diamond used for the first source test is a single $10 \times 10 \mathrm{~mm}^{2}$ diamond of $490 \mu \mathrm{m}$ thickness with a charge collection distance of $220 \mu \mathrm{m}$. Fig. 2 shows the average pulse shape of 1000 single pulses with amplitudes exceeding $1.0 \mathrm{mV}$. We observe diamond pulses with an average amplitude of $3.2 \mathrm{mV}$. The signal rise time is $780 \mathrm{ps}$ and the FWHM pulsewidth is $1.9 \mathrm{~ns}$ on average when measured on the amplifier output. The signal baseline is restored in less than $10 \mathrm{~ns}$ of the particle incident. From this measurement we can see that this FE-electronics combined with a pCVD diamond can fulfill the signal timing requirements of the BCM.

For reliable detection of single minimum ionizing particles in the $\mathrm{BCM}$ detector we aim at a higher signal-to-noise ratio (SNR) than the one achieved in the previous test. ${ }^{2}$ In order to increase the signal amplitude we investigate the simultaneous use of two diamonds acting as a single current source. The signals of two diamonds are passively summed before they are amplified. To achieved the summation, we mount a stack of two diamonds with identical acceptance area. The signal electrodes of the two diamond sides facing each other are shorted and connected to the amplifier. The two outside electrodes of the stack are connected to the bias voltage. In this configuration the full drift field of $2 \mathrm{~V} / \mu \mathrm{m}$ is applied to each diamond without further increase

\footnotetext{
${ }^{2}$ We define the SNR as the ratio of signal amplitude to r.m.s. noise.
} 


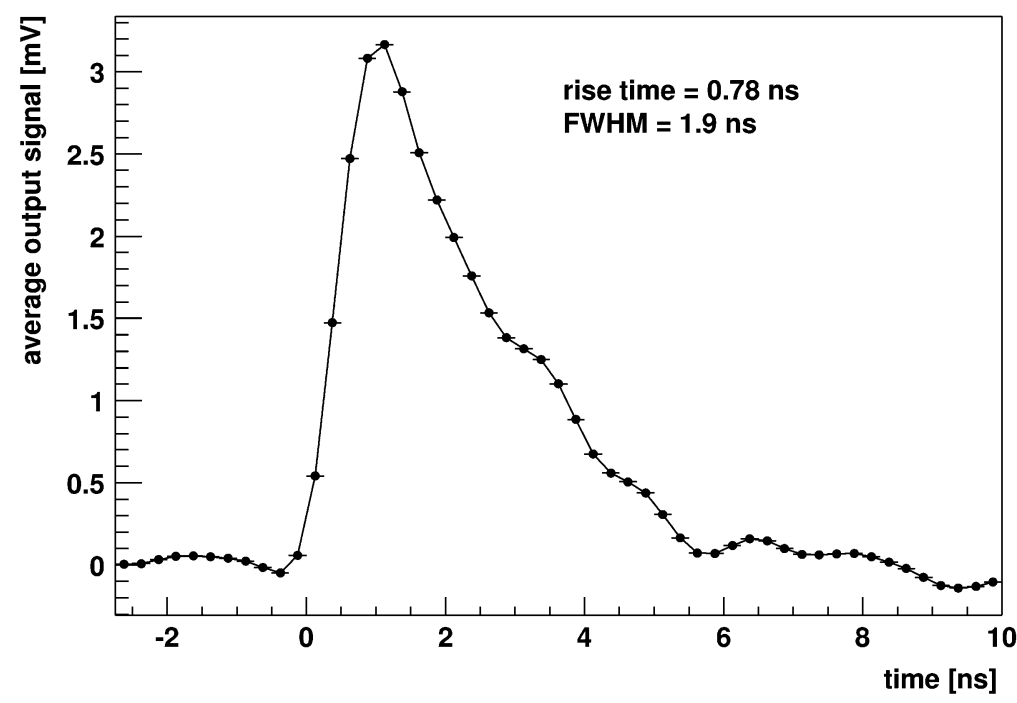

Fig. 2. Average signal of a single pCVD diamond coupled to the BCM FE-electronics during the source test.
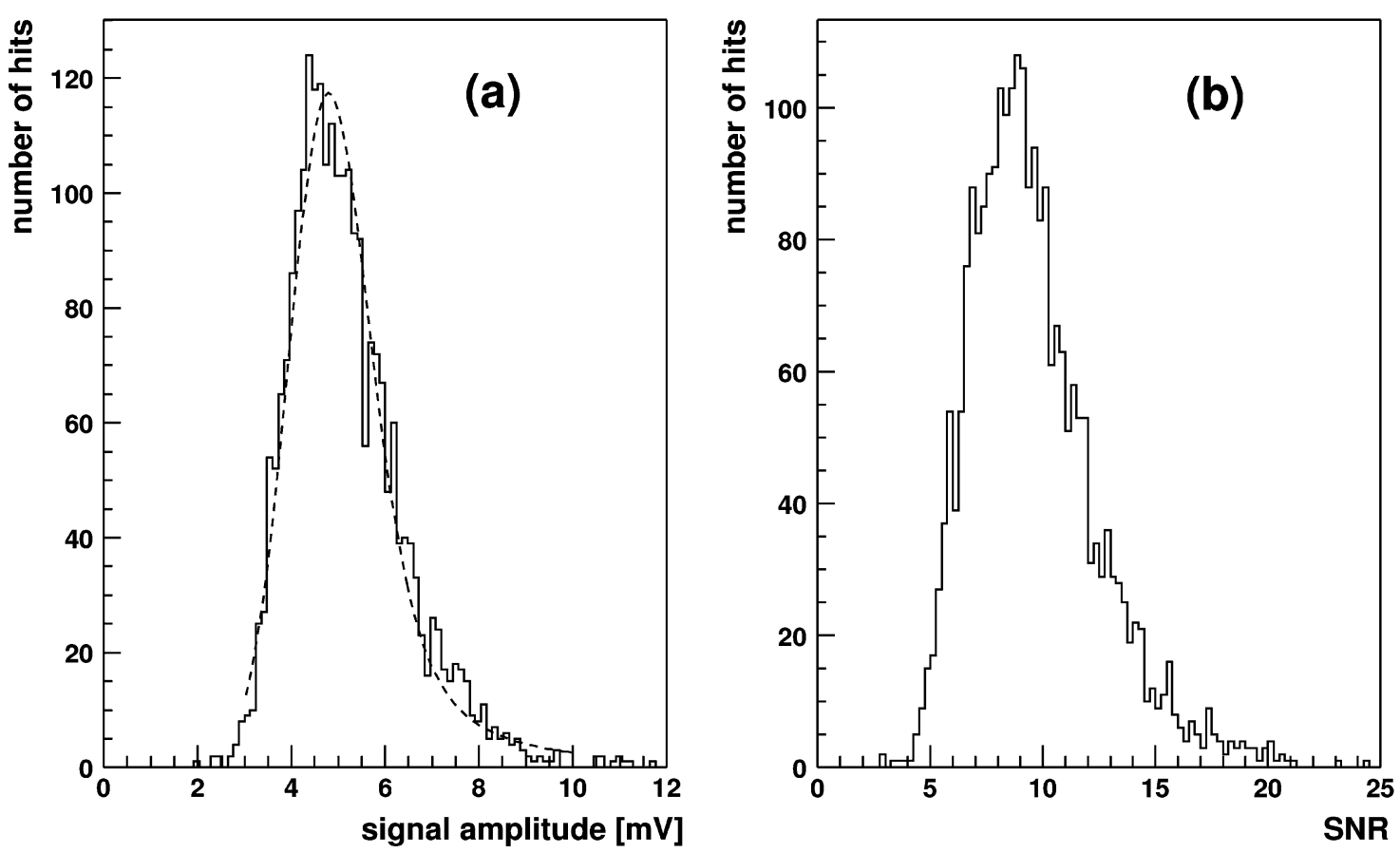

Fig. 3. Signal amplitude distribution (a) and signal-to-noise ratio (b) of the double-diamond assembly connected to the BCM FE-electronics during the source test.

of externally applied voltage. When a particle traverses the diamond stack, a separate ionization current pulse is generated in each diamond and the two current pulses are added on the common signal readout line. We further denote this assembly method as "double-diamond assembly."

A BCM test assembly has been constructed as double-diamond assembly using two $10 \times 10 \mathrm{~mm}^{2}$ pCVD diamonds of $360 \mu \mathrm{m}$ thickness with a charge collection distance of $160 \mu \mathrm{m}$ each. The double-diamond assembly was tested in the same setup as described above. Fig. 3(a) shows the amplitude distribution measured in the ${ }^{90} \mathrm{Sr}$-source setup on the double-diamond assembly. The average amplitude is found to be $5.2 \mathrm{mV}$, which is $60 \%$ higher than the signal previously measured on a single diamond. From a convoluted Landau-Gauss fit to the amplitude distribution, shown as dashed line, we estimate a most probable signal of $4.5 \mathrm{mV}$. The SNR distribution shown in Fig. 3(b) yields a most probable SNR of the double-diamond assembly of 8.3:1. When compared to the single-diamond assembly we observe a noise increase of approximately $15 \%$ and a rise-time increase of $120 \mathrm{ps}$ possibly due to the increase of effective detector capacitance with two diamonds on the amplifier input. Although the signal in the double-diamond assembly is significantly larger than for a single diamond, the measured increase does not match the expected factor of two. We attribute this to the use of different diamond substrates for double- and single-diamond assembly in our test, where the diamonds used for the double-diamond assembly were of slightly inferior quality and reduced thickness. In a later measurement [8] we could indeed confirm 

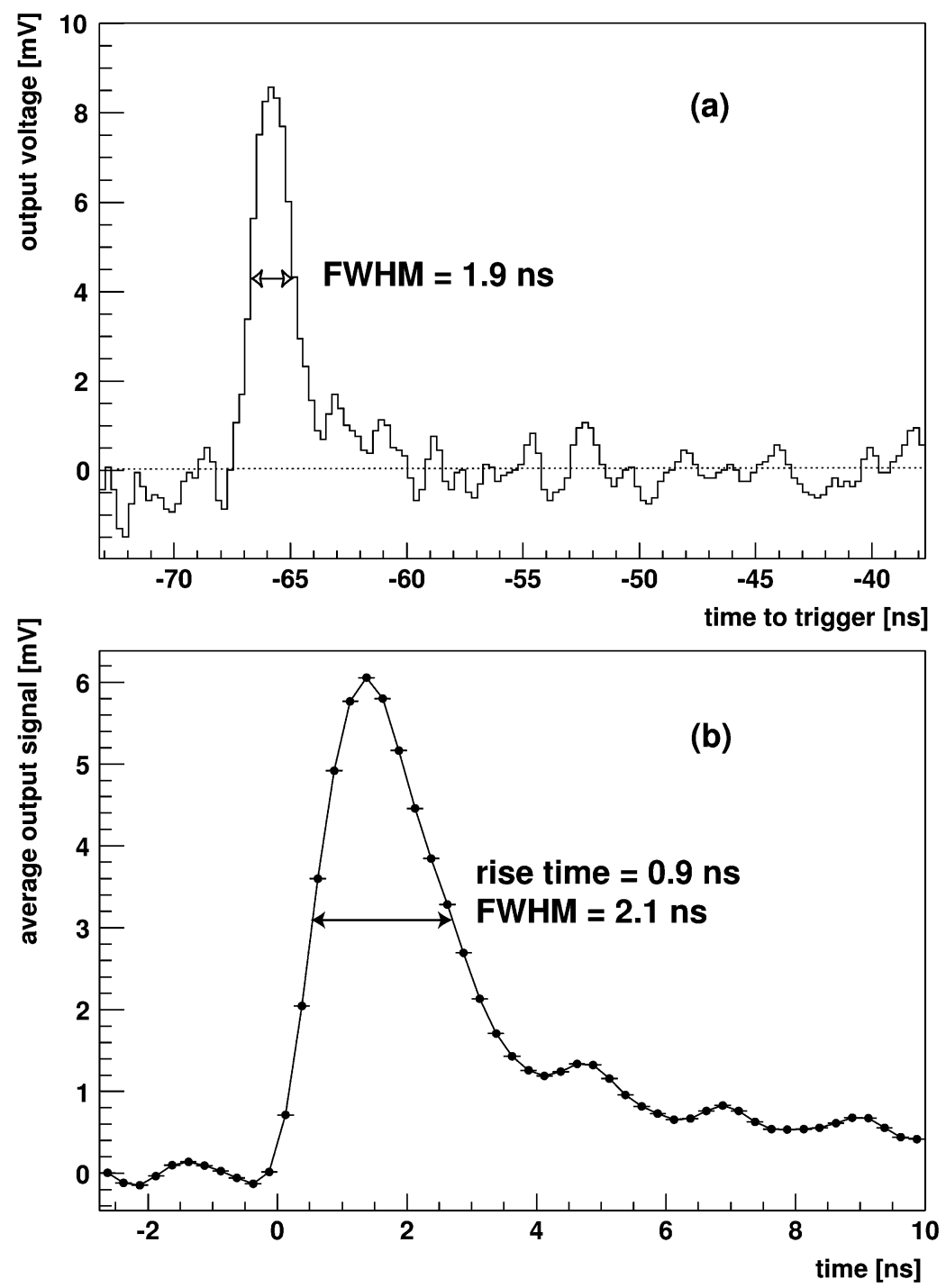

Fig. 4. Single pulse (a) and average signal (b) of the double-diamond assembly connected to the BCM FE-electronics in the beam test.

that the signal of a double-diamond assembly is twice the signal of a single diamond when identical diamond substrates are used.

\section{BeAm Test Results}

To evaluate the BCM response to single minimum-ionizing particles, we exposed a BCM test assembly to a $5 \mathrm{GeV} / \mathrm{c}$ pion beam at the CERN PS accelerator in May 2004. This test provided us with test conditions similar to the expected normal operation conditions in the ATLAS experiment. We tested the BCM double-diamond assembly described above in the configuration envisaged for the final BCM stations. The diamond assembly was placed at $45^{\circ}$ angle with respect to the pion beam. The FE-electronics is read out via a $16 \mathrm{~m}$ long coaxial cable to a digital oscilloscope. Individual BCM output signals are sampled at $4 \mathrm{Gs} / \mathrm{s}$ and stored for offline analysis. The scope readout is triggered by a scintillator which is aligned to the BCM diamond in the beam.

Fig. 4(a) shows a single MIP pulse recorded in the testbeam with a FWHM pulsewidth of 1.9 ns. Fig. 4(b) shows the average output pulse of the double-diamond assembly after $16 \mathrm{~m}$ of analog readout. On average we observe a rise time of 900 ps and a FWHM pulsewidth of 2.1 ns. For single events the pulsewidth varies from $1 \mathrm{~ns}$ to $3 \mathrm{~ns}$ for individual pulses due to the Landau fluctuation of the energy deposition and electronics noise. Fig. 4(b) shows that this prototype fulfills the signal speed requirements for the Atlas BCM.

The normalized amplitude distribution measured in the beam test is shown in Fig. 5 as hatched histogram. The amplitude distribution shows the expected Landau distribution with a most probable signal of $5.2 \mathrm{mV}$. The average amplitude is found to be $6.3 \mathrm{mV}$. For comparison the normalized noise distribution is shown on the same scale, which illustrates the clear separation of the signals from the noise. Fig. 6 shows the recorded SNR distribution for this data set. The BCM assembly shows a most probable SNR of 8.6:1 and an average SNR of 10.3:1.

\section{SUMMARY}

The ATLAS Beam Conditions Monitor is a high-speed beam monitor for measuring collision and background rates close to the interaction region. The detectors are polycrystalline CVD diamond detectors which are connected to high-speed current 


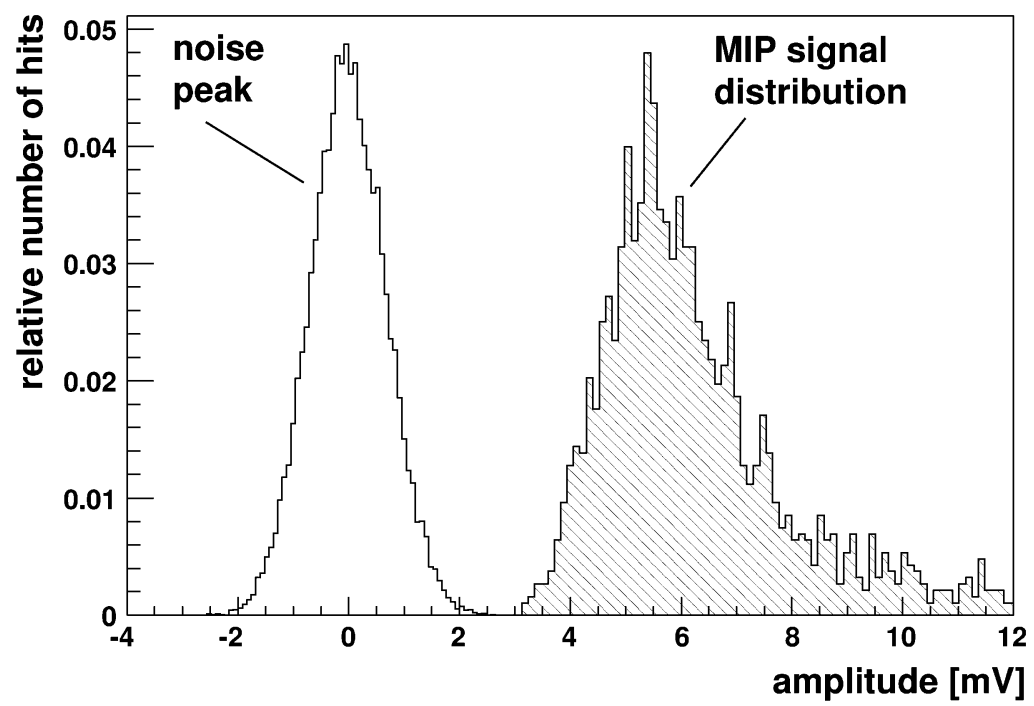

Fig. 5. Normalized signal amplitude distribution (hatched histogram) in comparison to noise distribution (open histogram) for double-diamond assembly in testbeam.

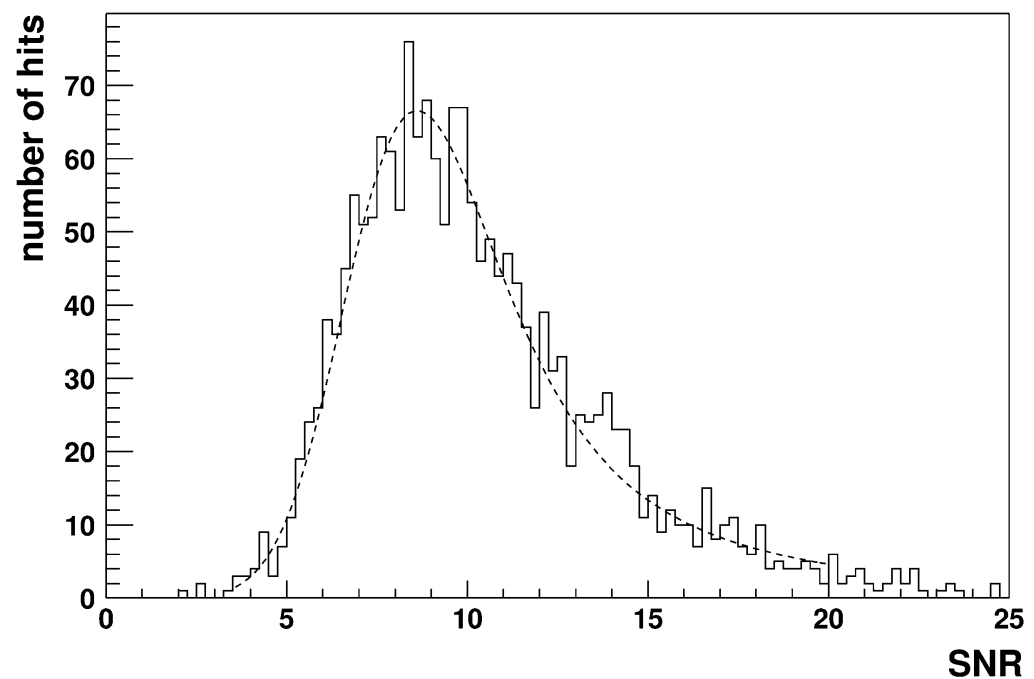

Fig. 6. Signal-to-noise distribution recorded in the beam test.

amplifiers for readout. The design has been optimized to be able to measure single minimum-ionizing particles with signal rise times of $1 \mathrm{~ns}$ and repetition rates of $\geq 100 \mathrm{MHz}$, which makes the detector applicable for the BCM. First prototype assemblies have been tested in $\beta$-source tests and a pion testbeam. The results have shown that we can achieve a reliable detection of MIPs and satisfy the challenging signal timing requirements using a double-diamond assembly. We were able to achieve signals with a mean rise time of 900 ps and FWHM pulsewidth of $2.1 \mathrm{~ns}$ after $16 \mathrm{~m}$ of analog readout cable. The recorded signal amplitude distribution follows the expected Landau distribution, the most probable signal-to-noise ratio was found to be 8.6:1 for single minimum-ionizing particles.

\section{ACKNOWLEDGMENT}

The author and the ATLAS Beam Conditions Monitor (BCM) Working Group would like to thank the members of the RD42 collaboration, FOTEC, and Rutgers University, Piscataway, NJ, for providing them with processed diamonds for this test. They also thank the members of the CMS Beam Conditions Monitor Group for their help during the preparation of the beam test.

\section{REFERENCES}

[1] The RD42 Collaboration, R\&D Proposal, Development of Diamond Tracking Detectors for High Luminosity Experiments at LHC, May 1994. DRDC/P56, CERN/DRDC 94-21.

[2] W. Adam et al., "Development of CVD diamond radiation detectors," Proc. Electrochemical Soc., vol. 97-32, pp. 491-497, 1997.

[3] — - "Radiation tolerance of CVD diamond detectors for pions and protons," Nucl. Instrum. Methods Phys. Res. A, vol. 476, pp. 686-693, 2002.

[4] S. Zhao, "Characterization of the electrical properties of polycrystalline diamond films," Ph.D. dissertation, Dept. of Physics, Ohio State Univ., Columbus, $\mathrm{OH}$.

[5] M. Franklin et al., "Development of diamond radiation detectors for SSC and LHC," Nucl. Instrum. Methods Phys. Res. A, vol. 315, pp. 39-42, 1992.

[6] H. Frais-Kölbl, E. Griesmayer, H. Kagan, and H. Pernegger, "A fast lownoise charged-particle CVD diamond detector," IEEE Trans. Nucl. Sci., vol. 51, no. 6, pp. 3833-3837, Dec. 2004.

[7] E. Berdermann, K. Blasche, P. Moritz, H. Stelzer, and B. Voss, "The use of CVD-diamond for heavy-ion detection," Diamond Related Mater., vol. 10, pp. 1770-1777, 2001.

[8] V. Cindro, Institute Jozef Stefan, Ljubljana, Slovenia, private communication, Nov. 2004. 